\title{
CONCEPT FOR AN INDUSTRIAL UBIQUITOUS ASSEMBLY ROBOT
}

\author{
Juhani Heilala*, Mikko Sallinen ${ }^{\S}$ \\ *VTT, P.O. BOX 1000, 02044 VTT, Finland, \\ Juhani.Heilala@vtt.fi \\ ${ }^{\S}$ VTT, P.O. BOX 1100,90571 Oulu, Finland \\ Mikko.Sallinen@vtt.fi
}

\begin{abstract}
We present a concept of a ubiquitous industrial robot. It has been defined to consist of technologies of artificial intelligence, ubiquitous computing, sensor network and industrial robots. The advantages compared with current intelligent robots are that they are more autonomous and they have cognitive skills. A ubiquitous robot is interoperable with all sensors, computers and other devices around it. One important factor is the natural interaction with humans. The ubiquitous approach is more common in consumer applications but still new in the industrial environment, even if many research efforts are being made. We also present an example of a ubiquitous robot: the isle of automation.
\end{abstract}

Keywords ubiquitous industrial robot, concept

\section{Introduction}

Manufacturing is facing growing challenges, and the global competition business environment is rapidly changing and filled with uncertainties. Typically, production is a high mix and low volume of customised personalised products. Production systems are also getting more complex due to advanced manufacturing technologies. Small lot sizes, versatility of product variations and shorter life cycles all set their own requirements. Production equipment must be fast to install and reconfigurable, and production set-up times must be short. In the flexible production of small lot sizes, human workers with their problem-solving abilities and cognitive capabilities are still the single best way to provide the required flexibility, adaptability and reliability. Cost competition and the need for productivity are forcing the trend towards automation. Another incentive for automation is the future likelihood of a lack of qualified personnel. The complexity of products sets further limitations and constraints on both automation and the skills and capabilities of human operators. Especially product miniaturisation has precision requirements that mean that some tasks are beyond the reach of the human worker.

The Preliminary Smart Assembly roadmap defined by the NIST workshop [1] identifies that empowered, knowledgeable people, a multi-disciplined, highly skilled workforce empowered to make the best overall decisions and working 
collaboratively with automation in a safe, shared environment for all tasks is part of the solution. The Manufuture Strategic Research Agenda 2006 [2] and the Technology Platform on Robotics, EUROP Strategic Research Agenda 2006 [3] both identify adaptive manufacturing as including the field of automation and robotics, robots as assistants of humans, hybrid assembly, and service robots. Adaptive manufacturing includes new automation solutions through the integration of new methods of cognitive information processing, signal processing and production control by high-speed information and communication systems.

In collaborative human centred automation, the main objective is to support human workers with qualified tools to increase productivity. The approach combines human creativity, intelligence, knowledge, flexibility, and skills as well as the advantages of sophisticated technical systems and tools, industrial cognitive robotics and efficient use of ICT (Fig 1). There are both physical and cognitive tasks that can and should be automated, as shown in Fig. 1. One key issue is to define the level of automation as discussed elsewhere [4].

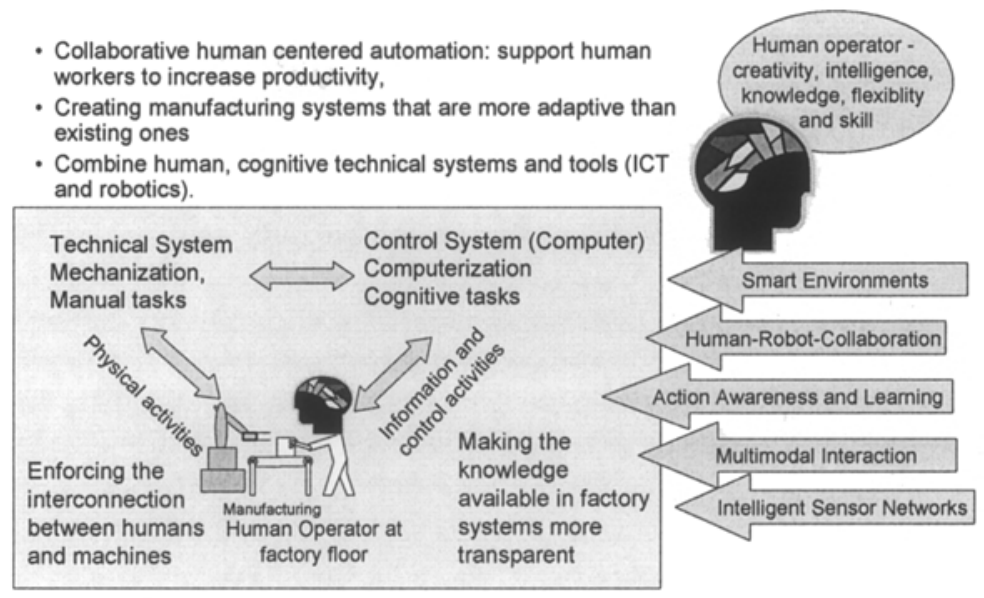

Fig. 1. Collaborative human-centred automation

A clear trend is emerging in the field of autonomous robotics, towards the integration of robotics with ambient intelligence and ubiquitous computing. This paper presents a concept for ubiquitous industrial robotics as well as future research needs. The concept combines an autonomous smart robot, networked smart production equipment, sensor networks and human collaboration in the intelligent assembly space.

\section{Concept for ubiquitous industrial robot workcell}

In recent years, due to the emergence of ubiquitous computing technology, a new class of networked robots called ubiquitous robots has been introduced. The networked robotic devices in smart environments, the ubiquitous robotics, provide a 
radically new way to build intelligent robot systems in the service of people [5]. Correspondingly, ubiquitous robot systems require a new way of thinking on the part of the designers of such systems, as well as new tools to build them.

The Ubiquitous Industrial Robot Workcell is our conceptual vision of ubiquitous service robots that provide the services the user needs on the factory floor. This kind of robotic system needs to be interoperable with sensors, sensor networks and devices in its current service environments automatically, rather than statically preprogrammed for its environment. Therefore artificial intelligence is needed [5]. The robot system must be autonomous, capable also of working with a human operator in the same smart or intelligent space (see Figs. 1, 2). In the industrial environment, since the environment is to some degree defined, fixed and known, a lot of autonomy can be built into the robot workcell even if many environment-, processand task-related inaccuracies or uncertain parameters remain.

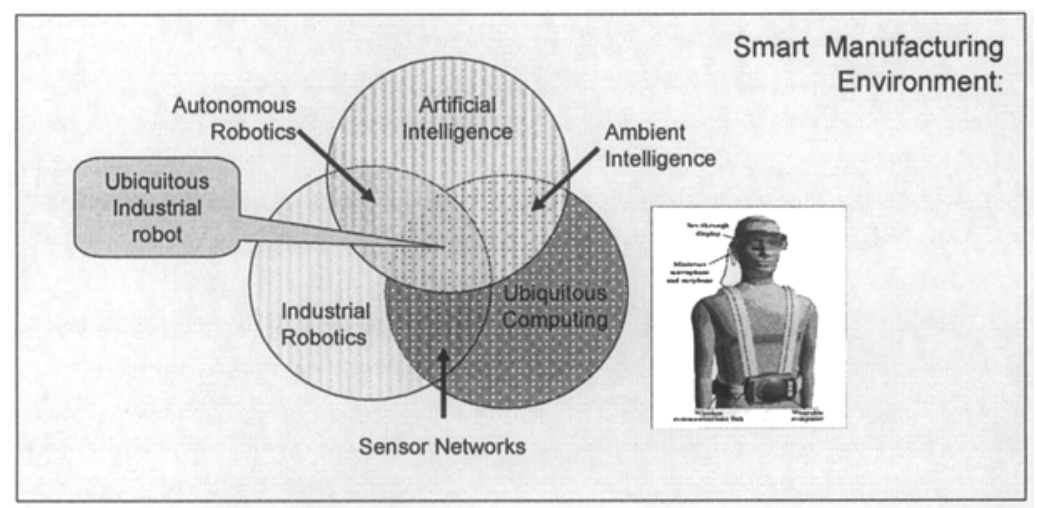

Fig. 2. The ubiquitous robot consists of three areas: Artificial intelligence, ubiquitous computing and industrial robotics, adapted from [5].

Typically, users' main tasks in automated, e.g. robot-based manufacturing systems are: configuring and teaching the system, monitoring and fine-tuning the system, charging and unloading, dealing with unplanned events, and communication with other team personnel. The general experience from the past is that the more advanced, complex and smarter the system is, the more critical is the role of the human operator [7], [8], [9]. Smart systems are vulnerable to failure and, therefore, they are even more dependent on human intervention than less intelligent ones. Usually the situation is the same if off-line programming is used in the system, especially when the lot sizes are small. Smart tools and artefacts should support users' normal behaviour, but more important is also managing an unpredictable situation; for example in the case of human error, the tools should be robust enough while being smart. They should help people manage complex situations, make good decisions, and act in a reasonable way.

A key element in flexible agile manufacturing is the ability to easily configure and program the systems concurrently, taking into account the relevant human factors and considering the change of systems and human activities in order to provide sufficient affordance. Several approaches have been developed to teach 
robots to perform complex actions. Some promising new approaches are based on learning by demonstration and learning by instruction paradigms, in which users first demonstrate the to-be-learned task or procedure [10,11, 12, and 13]. Important issues in this approach are: 1) understanding the procedure and the human intention; 2) learning and representing the procedure; and 3) mapping and executing the procedure with the system [13]. Knowledge of human-robot interaction plays a key role in the development of these systems.

\subsection{ICT for manufacturing and human operator}

One consequence of the deep penetration of ICT (Information and Communication Technology) into daily life is migration from the conventional factory floor to intelligent manufacturing environments built around the AmI (Ambient Intelligence) paradigm. That is, workplaces with emphasis on greater userfriendliness, more efficient service support, user-empowerment, and support for human interaction.

AmI has been defined [6] as "A concept in IST that presents what should come beyond the current "keyboard and screen" interfaces to enable ALL citizens to access IST services wherever they are, whenever they want, and in the form that is most natural for them. It involves new technologies and applications both for the access to, and for the provision of applications and services. It calls for the development of multi-sensorial interfaces which are supported by computing and networking technologies present everywhere and embedded in everyday objects. It also requires new tools and business models for service development and provision and for content creation and delivery." AmI is very similar to ubiquitous computing, since ubiquitous refers to "anywhere any time".

There are many needs for communication, as well as available technologies, in human-robot-smart artefact or smart manufacturing environment collaboration. Firstly, in the communication aspect, there is a two-tier communication environment: body area communication and operator-infrastructure communication. Body area communication can be carried out using RFID type communication, NFC (near field communication) or other short range communication technologies. In operator-infrastructure communication, WLAN, Bluetooth or GPRS communication can be used. It is important use as standard technologies as possible.

Communication can have many purposes. Sensor data can provide contextawareness and thus integration with manufacturing information systems, provide task support for operators, and the system can adapt to human operator needs. Operator hand movements, body gestures other measurements of physical actions can be used for the novel user interface. We can use either optical tracking, i.e. camera systems, or mechanical tracking with wearable motion sensors, force sensors or similar. 


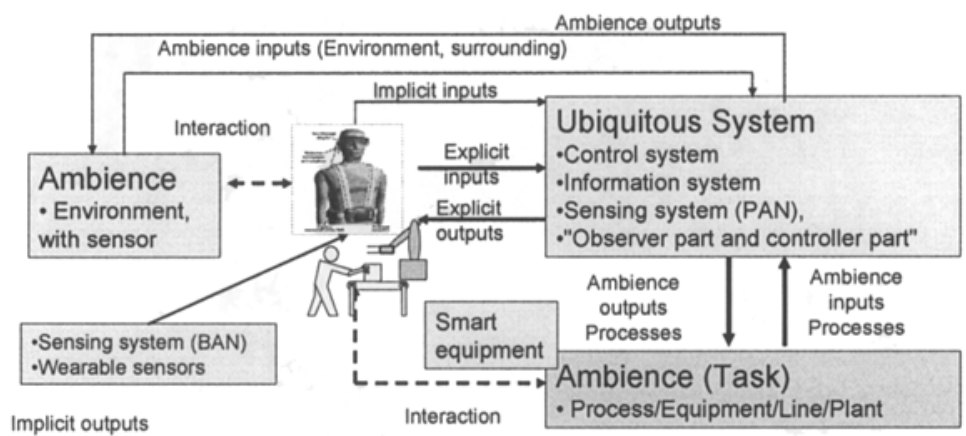

Fig. 3. A wearable, embedded computing system supports a human operating with a ubiquitous robot.

In the most advanced system, human operators carry a wearable, embedded system with sensors and local computing (see Fig. 3). The wireless body area network and sensors with localization and indoor positioning capability provide context and task awareness information even in a sensor-less environment. The industrial robot workcell is a smart environment; there are a lot of sensors for monitoring the manufacturing process and controlling the equipment.

\section{State of the art}

There are many initiatives for the development of ubiquitous robots, but they are mainly targeted for other areas than production [14, 15], such as service robots for the home and robot companions. Cognitive robotics is also a topic of EU focused research, including manufacturing applications. There are several EU-level largescale development projects focusing on collaborative industrial robots, SMERobot (www.smerobot.org) and PISA-IP (www.pisa-ip.org), just to mention a few. Industrial AmI is also being developed in several EU projects, but these are not dedicated to robotics.

The paradigm of the Cognitive Factory has been outlined by Zäh [16]. The Cognitive Factory is a factory environment with its machines, robots, storages, planning processes and human workers, that is equipped with a sensor network and an IT structure to allow the resources and processes to perceive what they are doing, enable them to control themselves and plan further actions in cooperation with other machines and human workers.

Acording to Vassos [17], cognitive robotics is a design paradigm that, when applied to robotic agents (in contrast to software agents), involves taking care of issues that lie in several different fields of research and applications, including:

1. The mechanical part of the robot that is responsible for movement and affecting the environment (e.g. mechanical arms, body, motors).

2. The software and hardware part responsible for getting meaningful information from the environment in which the robot is situated (e.g. the hardware and 
information processing software for doing feature extraction from visual images and sound).

3. The software part responsible for the representation of the environment and the way that the robot can interact with it (e.g. a logical specification of the properties of the environment as well as how these are affected by the available actions that the robot can perform).

4. The software part responsible for the specification of the task that the robot should do, based on the previous representation.

5. The software and hardware part that makes use of the representation of the environment and the specification of the task that the robot should do (numbers 3 and 4 ) in order to compute the behaviour of the robot at any given moment.

6. The software and hardware part that provides the interface between the reasoning component (number 5) and the actual sensors and actuators in the environment (numbers 1 and 2).

These guidelines can also be used for ubiquitous industrial robot system design.

\section{Example of a ubiquitous robot - the automation island}

An autonomous robotic workcell can be called a "production island". This island of automation provides a flexible way of producing short series production [18]. The concept of automation islands is modular and realises highly flexible and controllable robotised automation, and includes software components and hardware components operating synchronously. The basic element of the automation island is an industrial robot equipped with different kinds of sensors and auxiliary devices optimally combining mechanics, sensor technology and software. This gives highlevel flexibility in terms of programmability, reusability and price. The production system easily adapts to new products or product variants and to the deviation in work pieces.

The concept is an optimal combination of three technology elements: mechanics, sensor technology and software, as outlined in Fig. 4; the fourth element is the human operator and related interaction technology. Mechanics is utilised with clever design principles by applying low cost solutions whenever possible. The automation island is a part of the material flow process, from order to delivery. It is also part of the process from data file to work program and finally to the finished part. As a part of different processes and information flow, the automation island has appropriate contact points so that it is able to communicate and receive material and information. In addition, data acquisition presents new possibilities when open interfaces are offered up to sensor level. 


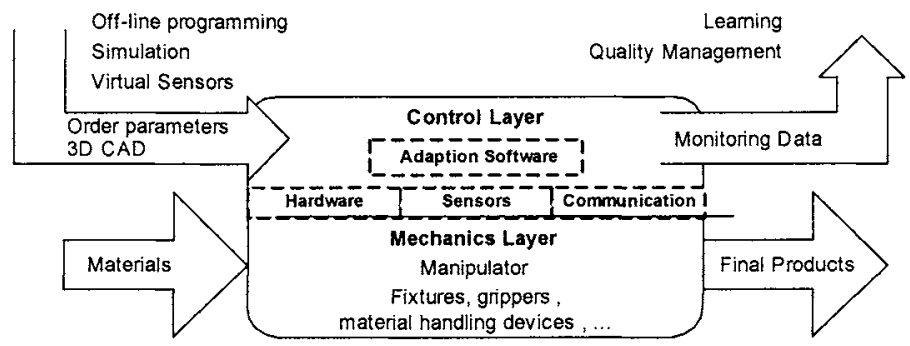

Fig. 4. Modular structure of the island of automation [18]

Interaction between the human operator and the island can be carried out in several ways. One solution is that the isle is making the measurements and provides processed results to the user, who makes the final decision. This approach provides an optimal fast solution to the control of production. Most of the need is required in the programming phase where information for manipulating a new object is fed to the system. In the future, robot systems will also include cognitive skills, which means that they learn from the tasks they are doing. Flexibility can be achieved using a multi-sensor system for observing the target object, advanced programming techniques, and a component-based approach for solving the application-related problems.

\section{Development needs}

A manufacturing environment where workforces are surrounded by a collection of reconfigurable production components (physical agents) that include mechatronics, control and intelligence (intelligent sensors and data processing units, autonomous, self-tuning and self-repair machines, intuitive multi-modal human machine interfaces, etc.) is an intelligent assembly space. In these circumstances, the challenge is to develop production automation and control systems with autonomy and intelligence or cognitive capabilities for co-operative/collaborative work, agile and fast adaptation to environmental changes, robustness against the occurrence of disturbances, and the easier integration of manufacturing resources and legacy systems. One of the major challenges is also human safety issues, which are not addressed in this article.

\section{Conclusions}

Recent manufacturing roadmaps are pinpointing the development needs in humansystem interaction, beyond the current HMI systems, and use of the keyboard as an input device and the display as the output device. In this paper, we present a novel system concept to be designed to fulfil the requirements for hybrid, knowledge- 
intensive manufacturing in the future, where humans and robots operate in close cooperation.

Related to the topic, the paradigm of the cognitive factory has been outlined by Zäh [16]. This is a factory environment with its machines, robots, storages, planning processes and human workers, that is equipped with a sensor network and an IT structure to allow the resources and processes to perceive what they are doing, enabling them to control themselves, and plan further actions in cooperation with other machines and human workers. It can be considered that the ubiquitous industrial robot concept introduced in this paper is one part of the cognitive factory.

\section{Acknowledgements}

The authors wish to acknowledge the financial support received from VTT. The development is part of the VTT Thema Complex System Design project KNOWMAN - Knowledge and Skill-Driven Manufacturing of High Added-Value Products.

\section{References}

1. Caie, J. ARC STRATEGIES. NIST Workshop defines preliminary Roadmaps for Smart Assembly. ARC Advisory Group, February 2007, 16 p.

2. MANUFUTURE - Strategic Research Agenda. Manufuture Technology Platform, September 2006. available from http://www.manufuture.org/strategic.html, accessed 13th July, 2007

3. EUROP - Strategic Research Agenda. Technology Platform on Robotics. May 2006, available from http://www,robotics-platform.eu.com/documents.htm accessed 13th July, 2007

4. Dencker, K., Stahre, J., Grondahl, P., Martensson, L., Lundholm, T., Johansson, C. An Approach to Proactive Assembly Systems: -Towards competitive assembly systems. IEEE International Symposium on Assembly and Manufacturing, ISAM '07. Ann Arbor, Michigan, USA, 22-25 July 2007, pp. $294-299$.

5. A. Saffiotti and M. Broxvall, "PEIS Ecologies: Ambient intelligence meets autonomous robotics," in Proc of the Int Conf on Smart Objects and Ambient Intelligence (sOcEUSAI), Grenoble, France, 2005, pp. 275-280.

6. http://istresults. cordis. europa.eu/index.cfm?section=overview\&tpl=glossary accessed 13 th July. 2007

7. Bainbridge, L. 1983. Ironies of automation. Automatica 19, 775-779.

8. Rasmussen, J. 1986. Information processing and human-machine interaction: An approach to cognitive engineering. New York: North-Holland. $215 \mathrm{p}$.

9. Vicente, K. J., \& Rasmussen, J. 1992. Ecological interface design: Theoretical foundations. IEEE Transactions on Systems, Man, and Cybernetics, 22, 4, p. 589-606.

10.Schraft, R. D., Meyer, C. The Need for an Intuitive Teaching Method for Small and Medium Enterprises. In: VDI-Wissensforum et al.: ISR 2006 - ROBOTIK 2006 : Proceedings of the Joint Conference on Robotics, May 15-17, 2006, Munich: Visions are Reality. Düsseldorf, 2006, 10 p. (CD-ROM), Abstract p. 95 (VDI-Berichte 1956). Available from www.smerobot.org - sientific publications. 
11.Barna Reskó, Andor Gaudia, Péter Baranyi, Trygve Thomessen. Ubiquitous Sensory Intelligence in Industrial Robot Programming. 5th International Symposium of Hungarian Researchers on Computational Intelligence. November 11-12, 2004, Budapes

12. Billard, A. \& Siegwart, R. 2004. Robot learning from demonstration. Robotics and Autonomous Systems 47, 65-67.

13. Dillman, R. 2004. Teaching and learning of robot tasks via observation of human performance. Robotics and Autonomous Systems 47, 109-116.

14.Kirchhoff, Uwe; Stokic, Dragan; Sundmaeker, Harald; AmI Technologies Based Business Improvement in Manufacturing SMEs. Paper at the eChallenges e-2006 Conference; 25 27 October, Barcelona, Spain.

15.Jong-Hwan Kim, Yong-Duk Kim, and Kang-Hee Lee. The Third Generation of Robotics: Ubiquitous Robot. 2nd International Conference on Autonomous Robots and Agents December 13-15, 2004 Palmerston North, New Zealand

16. Zäh, M. F.; Lau, C.; Wiesbeck, M.; Ostgathe, M.; Vogl, W. 2007. Towards the Cognitive Factory, in Proceedings of the 2nd International Conference on Changeable, Agile, Reconfigurable and Virtual Production (CARV 2007)

17. Stavros Vassos. Cognitive robotics in the industry. available at http://stavros.lostre.org/2007/05/14/cognitive-robotics-in-the-industry/ accessed $2^{\text {nd }}$ October, 2007

18. Sallinen, M.; Salmi, T.; Haataja, K.; Göös, J.; Voho, P. 2006. A Concept for Short Series Production and Manufacturing: Isles of Automation. Smart Systems 2006 \& ICMA 2006, Conference Proceedings (2006). 6th International Conference on Machine Automation (ICMA2006). Seinäjoki, 7 - 8 June 2006. 